\title{
POPULARIZING POOR MAN'S STERI-STRIP - WASH PROOF BAND-AID
}

Ramesh Babu Kㄹ, Naveen N², Kamal Kumar M³ , Prema Dhanraj ${ }^{4}$

\section{HOW TO CITE THIS ARTICLE:}

Ramesh Babu K, Naveen N, Kamal Kumar M, Prema Dhanraj. "Popularizing Poor Man's Steri-Strip - Wash Proof Band-Aid". Journal of Evolution of Medical and Dental Sciences 2014; Vol. 3, Issue 24, June 16; Page: 6790-6793, DOI: $10.14260 /$ jemds/2014/2820

ABSTRACT: Steri-Strips also known as 'Butterfly Stitches' are applied across the wound in such a manner so that the skin edges are approximated well and held together. They can be used instead of sutures and staples to avoid unsightly hatch marks and in patients who are not keen to undergo suturing or where the facilities are not available for a surgical closure. Rarely, they can also used to fix the spilt skin graft to the normal skin. However the limiting factor is the cost. Wash proof Band-Aid serves the purpose to the same extent. Band-Aid is covered by skin matching colored plastic, woven fabric with a felt pad in the centre containing antiseptic with a good absorption capacity. Our method of use of cost effective and easily available Band-Aids for sutureless wound closure showcases their easy application in day to day clinical practice.

KEYWORDS: Steri-Strips; Band-Aid; Sutureless wound closure.

INTRODUCTION: Steri-Strips (3M's Nexcare) ${ }^{1}$, generically known as 'Butterfly Stitches', are thin adhesive strips which can be used to close small wounds, . They are applied across the wound in such a manner so that the skin edges are approximated well and held together. They can be used instead of sutures and staples to avoid unsightly hatch marks and in patients who are not keen to undergo suturing or where the facilities are not available for a surgical closure. They are relatively easier to care for too. They are also used to avoid the gaping of the wound or wide scar formation following early suture or staple removal. They are also used to lessen the hypertrophic scarring. ${ }^{2}$

Rarely these Steri-Strips can also used to fix the spilt skin graft (stored skin application or delayed skin graft application in the ward) to the normal skin.

The Steri-Strips have the following qualities to serve us:

1. Easy and painless applicaton ${ }^{3}$

2. Reduced wound closure time

3. Reduced trauma to surrounding tissue-Hatch marks

4. Improved cosmetic results - hypertrophic scars are lessened 4

5. No risk of stitch abscess

6. Health economics - cheap \& saves working hours of patient 5

However the limiting factor is the cost. With the qualities of Steri-Strip but with less cost, wash proof Band-Aid serves the purpose to the same extent.

Band-Aid is covered by skin matching colored plastic, woven fabric with a felt pad in the centre containing antiseptic with a good absorption capacity. Some bandages have a thin, porous, non adherent film over the felt pad. 
HOW WE DO IT: Methods of application of Wash-Proof band aid to the wound:

First wounds are cleaned with Savlon / Betadine and washed with saline haemostasis secured, Benzion is applied on both sides of the wound and is allowed to dry so that the Band-Aid will be held firmly in place for longer duration.

I Method: Wash proof band aid plaster is obtained by peeling off from the pouch in a sterile technique. It is applied across the wound after removing the paper from the plaster. It is applied directly or after shaping or after cutting into small width plaster strips. The strips are pressed on to the benzoin applied skin (dried) on one side, margins are pulled to approximated the edges, dried if any discharge and fixed the other end of the plaster to the other side of wound either vertically or obliquely. The medicated pad is left exactly at the closed line of the wound, so that any discharge is absorbed and the antiseptic on the pad will lessen the wound infection.

In the centre at the felt pad area, the band aid is not adherent and hence it will allow the wound distraction forces to act which will result in a wide and hypertrophic scar. It also gives an ugly plaster appearance for a small wound.

II Method: Peel the paper on both sides of the plaster and cut the felt pad, so that we can use the both sides plastic film in the above manner. But it is very difficult to peel the plastic film sheet from the paper for use.

III Method: Here we cut first the wash proof band aid (taken from the pouch) from both ends as small strips of the width of the surgeon desire up to the felt pad. Now the paper position is slowly peeled off to see the side of the felt pad. We cut the felt pad on both side, so that we have 2 pieces from one wash proof Band-Aid. Now with Adson's forceps we can easily peel off the thin, narrow width skin colored plastic film strips from these pieces very easily for use.

It is narrow width, small size hence easily acceptable to the patient. The felt is not there in the centre, it holds the margins of the wound well to give a cosmetically acceptable scar.

III Method application in detail:

- Cut the plaster with the narrow width vertically from one end till the pad margin

- Repeat the procedure on the other side also

- Peel the paper from the plaster so that you can see the sides of the felt pad on both sides

- Cut the felt both sides, so that you get 2 pieces from Band-Aid

- Wound is prepared like the $1^{\text {st }}$ method

- Peel of the plastic film (small width) from one piece easily with Adson forceps (Non-toothed)

- Apply the strip on one side of the wound and press it so that it will be adherent onto the Benzoin applied area

- Pull the wound margin to approximate the edge

- $\quad$ Dry the wound

- Fix the other end of the film on to the other side of the wound

This procedure will give narrow width film of small sizes for easy application. It is much better to look; hence patient will accept and will comply for re-application if it is needed. Because it doesn't have the felt pad, it will hold the margins of the wound together resulting in a better cosmetic scar. 
This method can be used for closure of simple, fresh lacerated wounds and small avulsion injuries limited to skin and subcutaneous tissue. Though ideal to use in wounds less than $48 \mathrm{hrs,} \mathrm{it}$ also gives good results in late presenting patients who refuse to undergo surgical procedure (like in our patient in the photographs). Also useful in post-operative wound dehiscence. 6

We have been using this method for more than 15 years and it has been giving results as good as steri-strip. Hence we want to popularize this cheap, easy, painless wash proof band aid technique as a substitute to Steri-Strip.

\section{REFERENCES:}

1. '3M US: Nexcare Steri-Strip Skin Closure'. 3m.com

2. Murray, P.J.B.: Closure of Skin Wounds with Adhesive Tape, British Medical Journal, 2: 10301031, 1963.

3. Hirshman HP, Schuman DJ, Kajiyama G: Penetration of staphylococcus aureus into sutured wounds. J Ortho Res, 2: 269-271, 1984.

4. Traub, A. C. and Quattlebaum, F.W.: Cutaneous Wound Closure: Early Staple Removal and Replacement by Skin Tapes, Contemporary Surgery, 18: 1981.

5. Emmett, A.J.J., M.D., and Barron, J.N., M.D.: Adhesive Suture Strip Closure of Wounds in Plastic Surgery, British Journal of Plastic Surgery, 17: 175-183, 1964.

6. Pepicello J, Yavorek H: Five year experience with tape closure of abdominal wounds. Surgery Gynecology and Obstetrics, 1989, 169(4); 310-314.
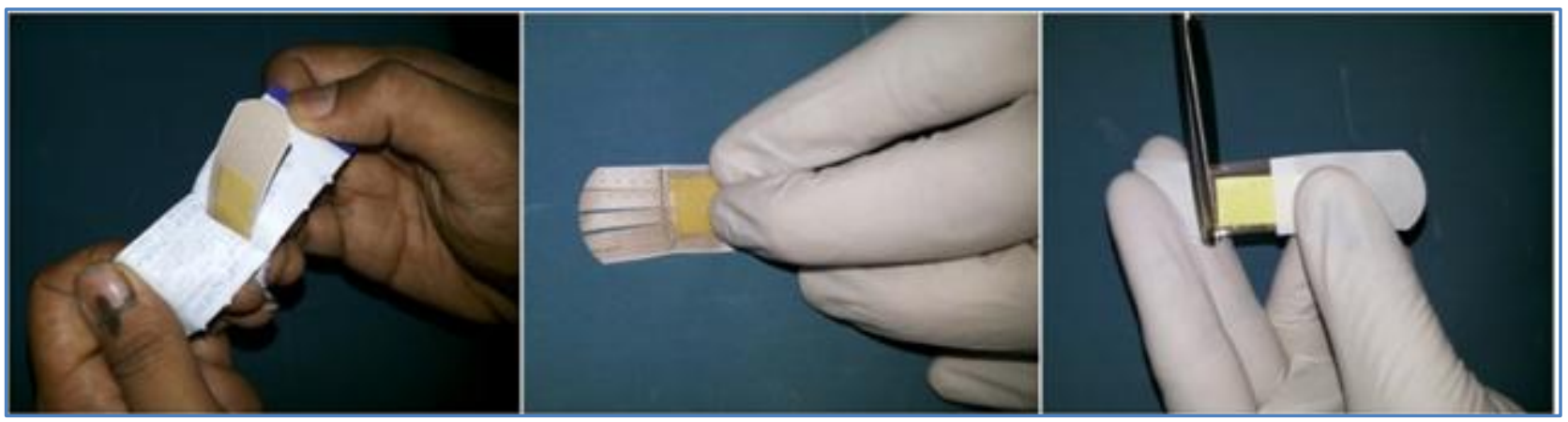

Fig. 1: Steps in Preparing Strips from Band-Aid
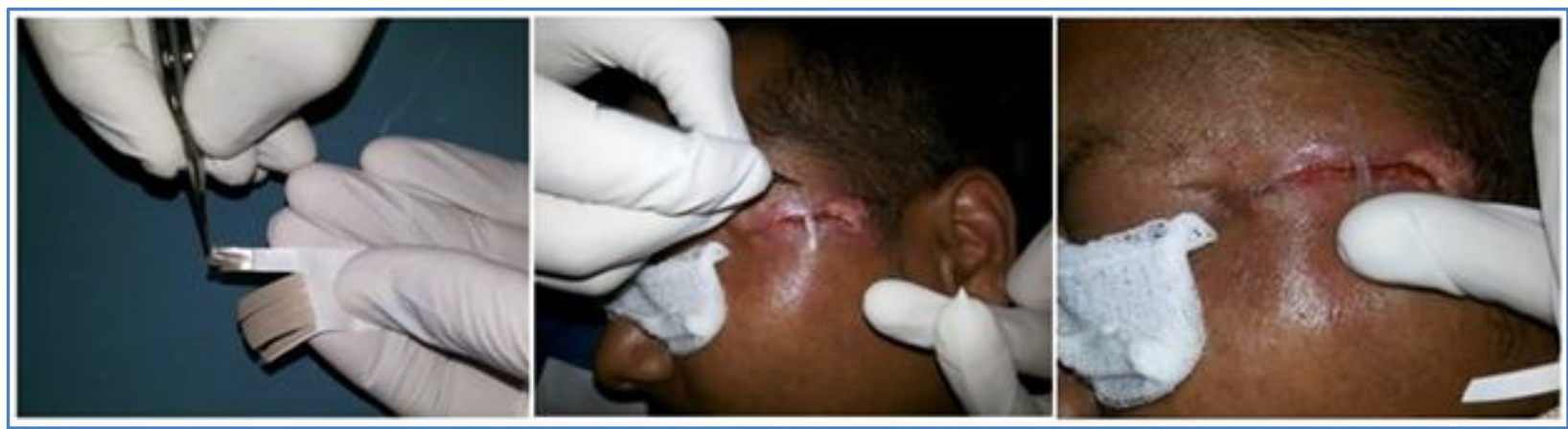

Fig. 2: Steps in Application of Band-Aid Strips in Approximating the Wound 


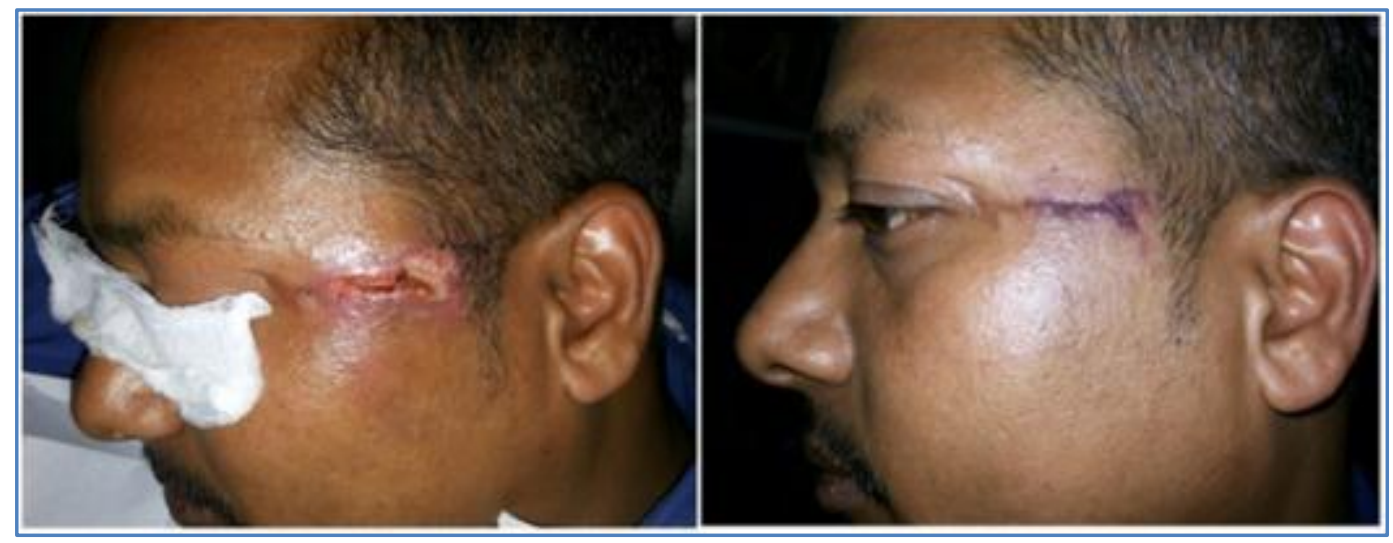

Fig. 3: This patient presented 5 days after trauma. Wound was oedematous with serous discharge. First picture is at the time of presentation and before Band-Aid closure. Second picture is $\mathbf{1 0}$ days after (Post Band-Aid removal)

\section{AUTHORS:}

1. Ramesh Babu K.

2. Naveen $\mathrm{N}$.

3. Kamal Kumar M

4. Prema Dhanraj

\section{PARTICULARS OF CONTRIBUTORS:}

1. Professor, Department of Plastic Surgery, Raja Rajeswari Medical College and Hospital.

2. Resident, Department of Plastic Surgery, Raja Rajeswari Medical College and Hospital.

3. Associate Professor, Department of Plastic Surgery, Raja Rajeswari Medical College and Hospital.

4. Professor and HOD, Department of Plastic Surgery, Raja Rajeswari Medical College and Hospital.

\section{NAME ADDRESS EMAIL ID OF THE CORRESPONDING AUTHOR:}

Dr. Ramesh Babu,

Professor, Department of Plastic Surgery,

Raja Rajeswari Medical College and Hospital,

Kambipura, Kengeri Hobli,

Mysore Road, Bangalore - 560074 .

E-mail: naveen_uno1@yahoo.co.in

Date of Submission: 18/05/2014.

Date of Peer Review: 19/05/2014.

Date of Acceptance: 07/06/2014.

Date of Publishing: 16/06/2014. 